\title{
COVID Feel Good: A Free VR Self-Help Solution for Providing Stress Management and Social Support During the COVID-19 Pandemic
}

\author{
Compiled by: Giuseppe Riva, $\mathrm{PhD}{ }^{1,2}$ and Eleonora Riva, $\mathrm{PhD}^{3}$
}

This column will try to describe the characteristics of current cyberpsychology research in Europe. In particular, CyberEurope aims at describing the leading research groups and projects running on the other side of the Ocean.

The coronavirus disease 2019 (COVID-19) pandemic and its social and economic consequences are having a major impact on psychological well-being, with side effects such as sadness, grief, irritability, and mood swings. Crucially, given the mandatory loneliness resulting from lockdown measures, easy-to-use, inexpensive, and scientifically validated selfhelp solutions are required.

In this regard, the COVID Feel Good (www.covidfeelgood .com) project is developing and testing a free weekly selfhelp virtual reality (VR) protocol to help overcome the psychological burden of COVID-19.

\section{An International Project}

COVID Feel Good is the result of work by several multidisciplinary researchers-psychologists and psychotherapists, 3D artists, musicians, storytellers, and designers - from around the world. Researchers from eight different countries, coordinated by Prof. Giuseppe Riva and Prof. Brenda K. Wiederhold, are exploiting the experiential and transformative potential of VR to provide stress management and social support using an easy self-help solution.

To reach this goal, the protocol will use VR to offer a natural digital place in which participants can relax and reflect. This effect will be enhanced by different daily social tasks aiming to facilitate a process of critical examination and eventually revision of core assumptions and beliefs.

However, the goal of the self-help protocol is not to provide a full structured psychological intervention. As underlined by Giuseppe Riva, "Our goal is to build the surgical mask of mental health support. Surgical masks do not provide the wearer with a reliable level of protection against coronavirus (20\%) versus the $95 / 99 \%$ of FFP2 and FFP3 masks. However, they are very effective in protecting others from the wearer's respiratory emissions, and their use is significantly better than wearing a scarf. With the project, we try to do the same. The goal of this VR protocol is not to solve complex mental health problems, but rather to reduce the burden of the quarantine by relieving anxiety and stress and improving interpersonal relationships."

\section{How to Try the VR Protocol}

For a long time, the main barrier to a broad use of VR technology was its cost. However, the developed protocol is free, and can be experienced using any smartphone with the YouTube app. The only technical requirement is the availability of VR glasses supporting the Google Cardboard format. These glasses, which can be found in online stores for US $\$ 10-20$, comprise nothing but a pair of magnifying lenses and a sheet of cardboard or a plastic box.

The protocol consists of watching a 10-minute VR video, named "The Secret Garden," at least once a day for a week. It is available in eight different languages: English, Spanish, French, Brazilian Portuguese, Italian, Catalan, Korean, and Japanese. More will be added in the coming months.

Participants need to watch the video using the VR glasses. Then, they need to follow a series of social exercises provided in the Web site project, with specific goals for each day of the week.

The aim of the first day is to prevent individuals from becoming obsessed with coronavirus. The second session aims to increase self-esteem. In the third session, the aim is to work on autobiographical memory (what we are and what we want). The fourth day aims to wake up the sense of community so that subjects do not feel alone. The fifth day works on taking back the dreams and goals that participants had before lockdown started. On the sixth day, the goal is to work on empathy, and on the last day, the aim is to plan a change.

All the exercises are designed to be experienced with another person (not necessarily physically together) in order to facilitate a process of critical examination and eventual

\footnotetext{
${ }^{1}$ Department of Psychology, Catholic University of Milan, Milan, Italy.

${ }^{2}$ Applied Technology for Neuro-Psychology Lab, Istituto Auxologico Italiano, Milan, Italy.

${ }^{3}$ Department of Cultural Heritage and Environment, University of Milan, Milan, Italy.
} 
revision of core assumptions and beliefs related to personal identity, relationships, and goals. Specifically, by facilitating self-reflectiveness and constructive exchange with relevant others, the protocol aims to improve our ability to adapt to the challenges provided by the pandemic.

According to Giuseppe Riva, "One of the paradoxes in this health crisis is that, despite being a problem, it can be a unique opportunity. Actually, whether we want or not, this forces us to change and manage new situations like quarantine, living with children and our partner, and a lack of relationships, among others. This tool can be useful not only to cope with a difficult situation but also to find new and unexpected resources to start again."

The project is now working on a multicentric controlled study in five countries to evaluate the efficiency of the protocol regarding the improvement of different psychological and well-being variables.
Address correspondence to: Dr. Giuseppe Riva Department of Psychology Catholic University of Milan Largo Gemelli 1-20123 Milan Italy

E-mail: giuseppe.riva@unicatt.it

Dr. Eleonora Riva Department of Cultural Heritage and Environment University of Milan Milan Italy

E-mail: eleonora.riva@unimi.it 